\title{
ELECTRONIC SPECKLE PATTERN INTERFEROMETRY FOR VIBRATIONAL ANALYSIS OF CUTTING TOOLS
}

\author{
Piotr MROZEK*, Ewa MROZEK**, Andrzej WERNER*
}

\author{
*Faculty of Mechanical Engineering, Department of Materials and Production Engineering, Bialystok University of Technology, \\ Wiejska 45C, 15-351 Bialystok, Poland \\ **Faculty of Mechanical Engineering, Department of Mechanics and Applied Computer Science, Bialystok University of Technology, \\ Wiejska 45C, 15-351 Bialystok, Poland \\ p.mrozek@pb.edu.pl, e.mrozek@pb.edu.pl, a.werner@pb.edu.pl
}

received 13 October 2017, revised 10 June 2018, accepted 13 June 2018

\begin{abstract}
A Michelson interferometer based ESPI system for static and vibration out of plane displacement measurements is presented. The aim of the article is to demonstrate the usability of ESPI non-contact measurement method in the field of machining. The correlation fringe patterns were visualized using custom software. The accuracy of ESPI interferometer was verified by the comparison with measurement results collected using industrial XL- 80 laser system. The efficacy in vibration analysis was tested by studying the mode shapes and resonant frequencies of the transverse vibrations of square plates. The measurement methodology was used to determine natural frequencies and the shapes of vibrational modes of NFTe 100x1.2/64-II circular slitting saw. As a result the values of rotational speed that should be avoided during machining were determined.
\end{abstract}

Key words: Interferometry, Speckle Pattern, Correlation Fringes, Vibrational Analysis

\section{INTRODUCTION}

Advanced optical measurement methods, due to their high sensitivity and non-contact character, have been widely accepted both in scientific research and industry as a tool for displacement and vibrations analysis. Electronic speckle pattern interferometry (ESPI) is the most sensitive and accurate optical method for fullfield three-dimensional (3D) displacement assessment ( $\mathrm{Fu}$ and Moosa, 2002; Hild and Roux, 2006; Yang et al., 2014). In experimental non-contact vibration analysis time-averaged amplitude fluctuation (AF)-ESPI is the most widely used optical method. The advantage of AF-ESPI method is that both resonant frequencies and the corresponding mode shapes for transverse (out of plane) modes can be obtained. Experimentally revealed correlation fringe patterns of AF-ESPI correspond to the natural vibrational modes. So far, several different ESPI systems have been commercialized (Dantec Dynamics, Laser Technology Inc.), nevertheless due to significant progress made in corresponding fields of modern technology: laser sources, computer equipment and high resolution CCD and CMOS detector arrays, a variety of robust custom constructions of ESPI systems have been worked out by different research groups (Augulis et al., 2004; Quin et al., 2016). ESPI has been applied to experimentally verify the analytical and numerical models of applied mechanics (Halama et al., 2016), vibration theory (Chi-Hung et al., 2004), in automotive industry (Beeck and Hentschel, 2000; Ven der Auweraer et al., 2002), materials engineering (Richardson et al., 1998), biomedical area (Yang et al., 2014), micro-electro-mechanical systems (MEMS) (Foitzik et al., 2003) and in many other fields of science and engineering.

A field in which knowledge of frequencies of natural vibrations and natural vibrational modes plays a significant role is mechani- cal engineering and the engineering of cutting tools (Rothberg and Bell, 2004; Tatar and Gren, 2008). Vibrations of tools and components of cutting machines directly affect machining accuracy, geometric structure of the product surface and the noise generated during machining. Until now relatively little attention has been paid to experimental research of vibrations of cutting tools using AF-ESPI. In the present study a Michelson interferometer based ESPI system for static and vibration out of plane displacement measurements is presented. The instrument was built using components actually available as standard. Custom software was worked out to visualize the correlation fringe patterns. The accuracy of the device was verified by the comparison with measurement results collected using industrial laser interferometer and the efficacy in vibration analysis was tested by studying the mode shapes and resonant frequencies of the transverse vibrations of square plates. A usefulness of the measurement methodology in the field of machining was revealed by the determination of natural frequencies and the shapes of vibrational modes of a circular slitting saw. The results may help to select the appropriate parameters of machining in order to minimize tool vibrations affecting roughness of the machined surface and noise generated during machining.

\section{MATERIALS AND METHODS}

\subsection{Michelson ESPI interferometer construction}

A schematic diagram of the setup of a Michelson interferometer based ESPI system is shown in Fig. 1. The subassemblies of the system were fixed to a massive cast iron frame to minimize 
the magnitude of eventual vibrational noise affecting the measurement accuracy. A light beam of He-Ne laser source 1 (12 mW, $\lambda=632.8 \mathrm{~nm}$ ) was diverged by a glass lens 2 and fell on a beam splitter 3 . The beam was divided into two equal intensity coherent beams, which illuminated two surfaces: $S_{0}$ (object surface 4 ) and $S_{r}$ (reference surface 5). The surfaces were coated with thin $(0.05$ $\mathrm{mm}$ thickness) white color light diffusive self-adhesive foil. Speckle patterns were generated by these surfaces. The speckle patterns after passing through a beam splitter and the camera 6 lens met and interfered with each other in the image plane of a CCD sensor of the camera (1/3" SONY EXview HAD CCD II). The aperture of the camera lens was fitted to adjust the size of the speckle to the size of a pixel of the CCD array. The average size of a speckle was slightly greater than the diagonal length of a pixel of the sensor. The relative intensities of the two beams were adjusted by the arrangement of $S_{0}$ and $S_{r}$ surface position. The exposure time of the camera was adjusted to acquire an image without saturating the CCD sensor. Bitmap files of 768x576 resolution were acquired by a video capture card installed in a PC. Deformations were applied to $S_{0}$ surface with $S_{r}$ undisturbed surface serving as a reference. An initial speckle pattern image was acquired when no extortion was applied to $S_{0}$ surface. Subtraction of an actual speckle pattern image from the initial speckle pattern image was accomplished by a custom software. The resulting correlation fringes were displayed on a $\mathrm{PC}$ monitor screen and transferred to the hard disc of PC with $40 \mathrm{~Hz}$ frequency. For a static deformation of $S_{0}$ surface a stationary correlation fringe pattern was obtained as a result of an actual from the initial speckle images subtraction. In a case of natural vibration analysis a loudspeaker driven by a function generator and an acoustic amplifier was used as the source of an excitation wave. When a vibrating frequency was in the neighborhood of the resonant frequency of object surface $S_{0}$, stationary correlation fringes were observed. The fringes became the most clear and intensive as the resonant frequency was approached. The resonant frequencies and corresponding mode shapes could be determined at the same time.

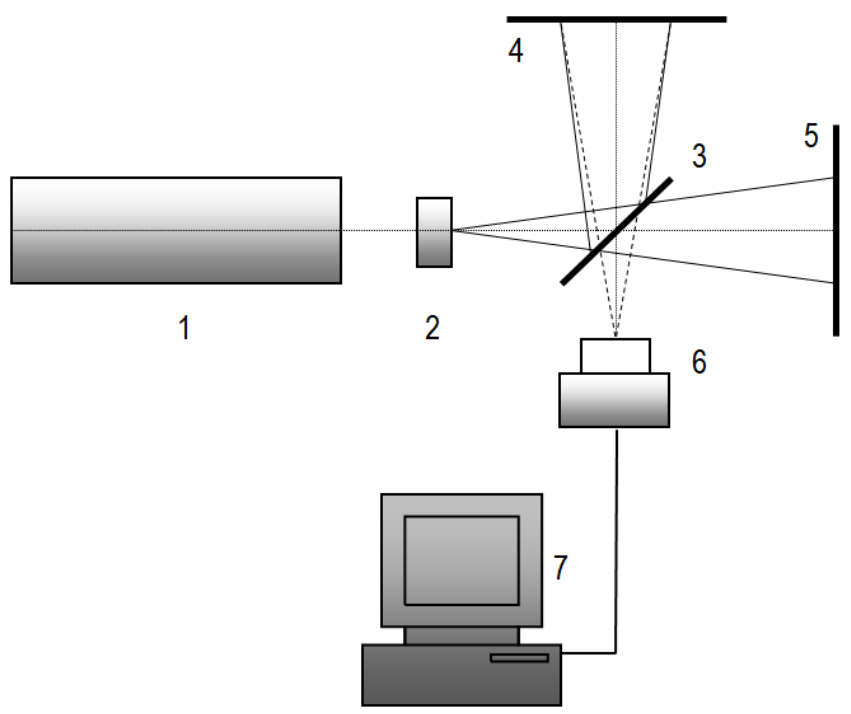

Fig. 1. Schematic diagram of ESPI setup: 1 - He-Ne laser, 2 - lens, 3 - beam splitter, 4 - object surface $S_{0}, 5$ - reference surface $S_{r}, 6$ - CCD camera, 7 - PC with a video capture card and a custom software for speckle patterns processing

\subsection{Basic principle of ESPI out of plane displacement measurements}

The wavefronts of the object $\left(U_{0}\right)$ and reference $\left(U_{r}\right)$ beam reflected from $S_{0}$ and $S_{r}$ surfaces (Fig. 1), respectively, can be expressed as (Yang et al., 2014):

$U_{0}=a_{0} \sin \left(\omega t+\varphi_{0}\right)$

$U_{r}=a_{r} \sin \left(\omega t+\varphi_{r}\right)$

where $a_{0}$ and $a_{r}$ are amplitudes of electric field strength, $\omega$ is an angular frequency of a light wave, $\varphi_{0}$ and $\varphi_{r}$ represent the phase of the object beam and the reference beam, respectively (Yang et al., 2014). $U_{0}$ and $U_{r}$ are functions of plane $x, y$ coordinates. Intensity $I$ of the speckle pattern image acquired by the CCD sensor can be written as:

$I=a_{0}^{2}+a_{r}^{2}+2 a_{0} a_{r} \cos \left(\varphi_{0}-\varphi_{r}\right)$

The argument in parentheses in Eq. (3) can be described as a phase difference:

$\Theta=\varphi_{0}-\varphi_{r}$

Eq. (3) can be written as:

$I=I_{0}(1+\gamma \cos \Theta)$

where:

$I_{0}=a_{0}^{2}+a_{r}^{2}$

is the background intensity and

$\gamma=\frac{2 a_{0} a_{r}}{a_{0}^{2}+a_{r}^{2}}$

is the contrast. When surface $S_{0}$ is deformed (with $S_{r}$ surface undisturbed), the intensity of the speckle pattern becomes:

$I=I_{0}(1+\gamma \cos (\Theta+\Delta))$

where $\Delta$ represents the phase difference shift of the speckle pattern before and after $S_{0}$ deformation:

$\Delta=\varphi^{\prime}{ }_{0}-\varphi_{0}$

For a Michelson-type interferometer the relationship between phase change $\Delta$ and displacement w of surface $S_{0}$ can be written as:

$\Delta=\frac{4 \pi}{\lambda} w$

For $\Delta=2 \pi$ which corresponds to transition between the centers of two adjacent fringes of order $i$ and $(i+1)$ in a correlation image, from Eq. (10) one can obtain:

$w=\frac{\lambda}{2}$

Eq. (11) defines a basic sensitivity of the correlation fringe pattern ESPI system. For a wavelength $\lambda=632 \mathrm{~nm}$ (He-Ne laser source) the basic sensitivity equals to $316 \mathrm{~nm}$.

\subsection{Basic expressions for natural frequencies of vibrating plates}

The equation of free motion for small out of plane deformations $w$ of a flat uniform elastic plate of density $\rho$, in $x, y$ 
Cartesian coordinates has the form (Blevins, 2016):

$\frac{E h^{3}}{12(1-v)}\left(\frac{\partial^{4} w}{\partial x^{4}}+2 \frac{\partial^{4} w}{\partial x^{2} y^{2}}+\frac{\partial^{4} w}{\partial y^{4}}\right)+\gamma \frac{\partial^{2} w}{\partial t^{2}}=0$

where: $E$ - modulus of elasticity of plate material, $v$ - Poisson's ratio, $h$ - plate thickness, $t$ - time, $\gamma$ - mass per unit area of the plate $(\gamma=\rho h)$.

Using a separation of variables solution for Eq. (12) allows to obtain the associated formulas of natural frequencies and mode shapes for rectangular and annular plates (Blevins, 2016). Natural frequency $f_{i j}$ is expressed in a form:

$f_{i j}=\frac{\lambda_{i j}^{2}}{2 \pi a^{2}}\left[\frac{E h^{3}}{12\left(1-v^{2}\right)}\right]^{\frac{1}{2}} \mathrm{~Hz}$

where: $i$ - number of half-waves in mode shape along $x$ axis (rectangular plate) or number of nodal diameters (round and annular plate), $j$ - number of half-waves in mode shape along $y$ axis (rectangular plate) or number of nodal circles (round and annular plate), $\lambda_{i j}$ - dimensionless frequency parameter, $a$ - linear dimension of the plate (length of a rectangular plate or outer radius of round or annular plate).

The mode shapes of rectangular plates are combinations of sinusoidal and hyperbolic functions. The mode shapes of circular plates are Bessel functions.

\section{EXPERIMENTAL}

\subsection{Verification of ESPI interferometer accuracy for static out of plane displacement measurement}

A general view of the experimental stand is shown in Fig. 2. A front surface of a rectangular $2 \mathrm{~mm}$ thick aluminum plate of dimensions $9 \times 10 \mathrm{~cm}^{2}$ (width and height, respectively) clamped at its bottom edge was used as $S_{0}$ surface (Fig. 3). A surface of $15 \mathrm{~mm}$ thick aluminum rectangular plate was used as a reference $S_{r}$ surface. At the upper edge of the back (opposite to $S_{0}$ surface) side of the plate, a retroreflector of Renishaw XL-80 laser measuring system was attached. A laser head and the interferometer of the XL-80 system were placed in front of the retroreflector. Slowly increasing load force was applied at the middle of the upper edge of the plate using a tensioned spring. A laser beam reflected from the retroreflector was used by the $\mathrm{XL}-80$ measuring system to evaluate the displacement of the retroreflector. Slight bending of the plate resulted in a decrease of a distance of $S_{0}$ to a laser head of XL-80 system and increase of a distance to $S_{r}$ surface. In this way a measurement of out of plane displacement of a central point of the retroreflector and the corresponding point of $S_{0}$ surface was possible at the same time using XL-80 system and ESPI interferometer, respectively. Due to Eq. (11) out of plane displacements $w_{i_{-} \text {ESPI }}$ of the points located at the center of a dark correlation fringe of $i$-th order can be expressed as:

$w_{i \_ \text {ESPI }}=i \frac{\lambda}{2}$

ESPI correlation fringes of increasing order $\mathrm{i}, \mathrm{w}_{\mathrm{i} \text { ESPI }}$ measurement results determined using Eq. (14) for $\lambda=632 \mathrm{~nm}$ and the corresponding values of $\mathrm{XL}-80$ system point displacement measurement results $\mathrm{w}_{\mathrm{XL} 80}$ for a central point of a retroreflector ("+" mark) for increasing deflection of the plate are shown in Fig. 4.

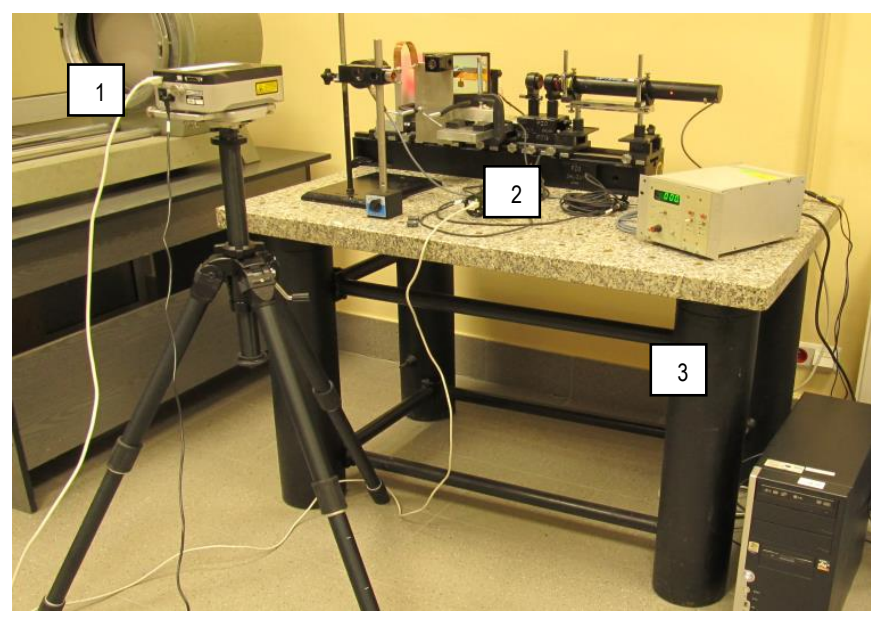

Fig. 2. A general view of the experimental setup: 1 - laser head of $X L-80$ system, 2 - ESPI interferometer, 3 - anti vibration table

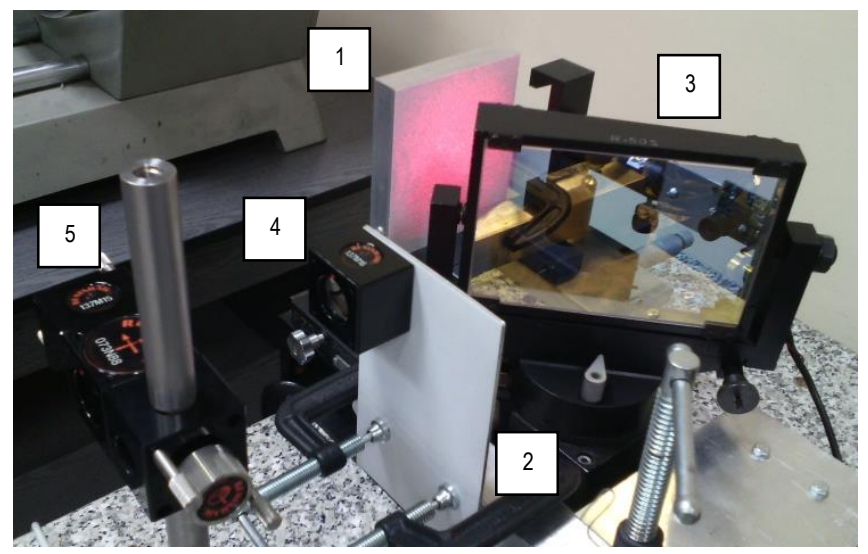

Fig. 3. A setup for verification of ESPI interferometer accuracy: 1 - reference plate, 2 - object plate, 3 - beam splitter, 4 - retroreflector of XL-80 system, 5 - optics of XL-80 system

\subsection{Experimental determination of natural frequencies and mode shapes of a vibrating square plate}

Resonant frequencies and corresponding mode shapes of the transverse vibrations were experimentally determined for square $9 \times 9 \mathrm{~cm}^{2}$ aluminum $2 \mathrm{~mm}$ thick plate. The plate was clamped at the bottom edge. Vibrations of the plate were excited by a louder driven by an amplifier and a sine wave function generator. The louder was located a short distance from the back side of the plate. When the frequency of vibration was equal to the resonant frequency, stationary and clear correlation fringe patterns were observed. The mode shapes for corresponding resonant frequencies are shown in Fig. 5. Dark correlation fringes represent nodal lines of mode shapes in the pictures.

\subsection{Experimental determination of natural frequencies and mode shapes of a vibrating circular slitting saw}

Resonant frequencies and corresponding mode shapes of the transverse vibrations of a circular slitting saw (NFTe 100x1.2/64II) were determined using a method described in Section 3.2. A slitting saw of $100 \mathrm{~mm}$ diameter and $1.2 \mathrm{~mm}$ thickness was used in the experiment (Fig. 6). The tool was clamped at the sides of a socket ( $30 \mathrm{~mm}$ diameter). The mode shapes for corresponding resonant frequencies are shown in Fig. 7. 

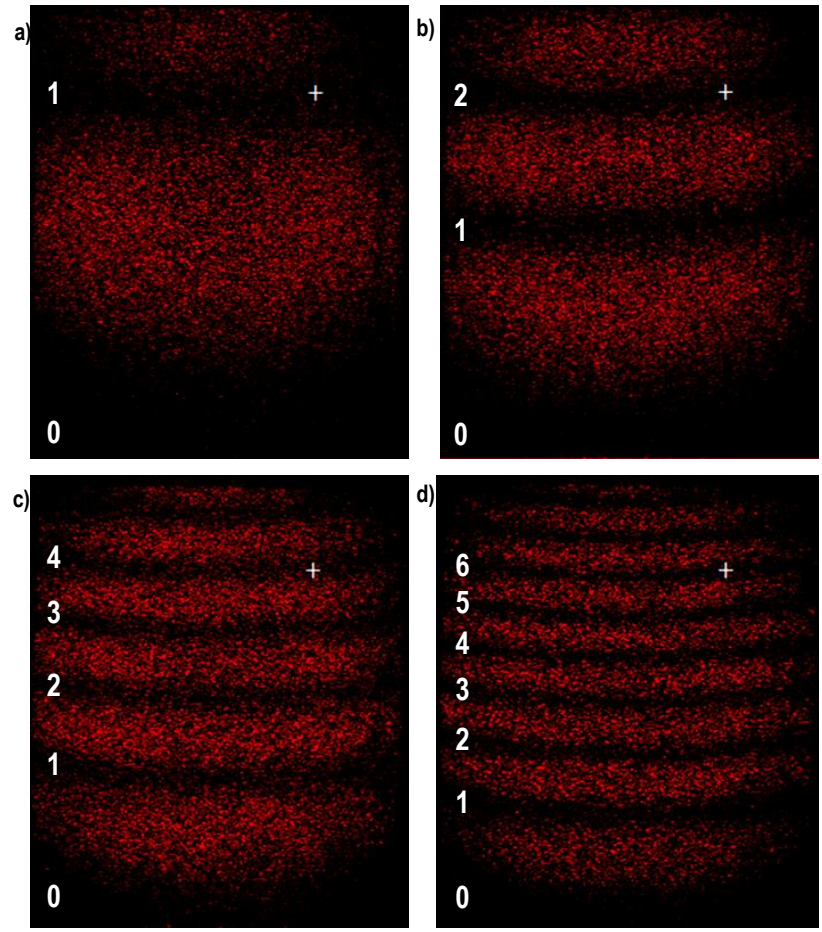

Fig. 4. ESPI correlation fringes for out of plane static displacements $w$ of a rectangular Al plate; the numbers at the left side of the picture represent the order $i$ of dark ESPI correlation fringes; "+" mark represents the position of a center of XL-80 system retroreflector used to measure $W_{X L-80}$ point displacement; ESPI and corresponding XL-80 measurement results: a) $w_{1} E S P I=0.316 \mu \mathrm{m}, w_{X L 80}=0.313$ $\mu \mathrm{m}$, b) $\left.w_{2 \_} E S P I=0.632 \mu \mathrm{m}, w_{X L 80}=0.627 \mu \mathrm{m}, \mathrm{c}\right) W_{4 \_E S P I}=1.266 \mu \mathrm{m}$, $W \times L 80=1.266 \mu \mathrm{m}, \mathrm{d}) W_{6} E S P P=1.896 \mu \mathrm{m}, w \times L 80=1.945 \mu \mathrm{m}$
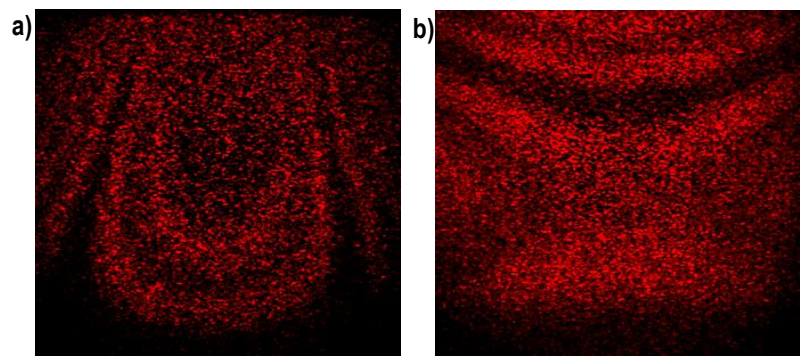

Fig. 5. Mode shapes of vibrations of a square Al plate at resonant frequencies: a) $f=532 \mathrm{~Hz}$, b) $f=390 \mathrm{~Hz}$

\section{RESULTS AND DISCUSSION}

Linear measurement accuracy of $\mathrm{XL}-80$ laser system is \pm 0.5 ppm due to a stabilised laser source and accurate environmental compensation (Brochure: XL-80 laser measurement system). In the experiment the distance between the retroreflector and the interferometer optics was $0.1 \mathrm{~m}$, therefore the static displacement of $S_{0}$ surface was measured with about $50 \mathrm{~nm}$ accuracy, which was higher than the basic sensitivity of the ESPI system described by Eq. (11). For the correlation fringes shown in Fig. 4. the dark fringes can be easily distinguished from the bright ones, therefore it seems that the accuracy of about $\lambda / 4$ (158 nm for He-Ne laser source) may be assumed for ESPI measurements in this case. The results of ESPI measurements show good agreement with $\mathrm{XL}-80$ assessment results, which may serve as the calibration data for the speckle interferometer.

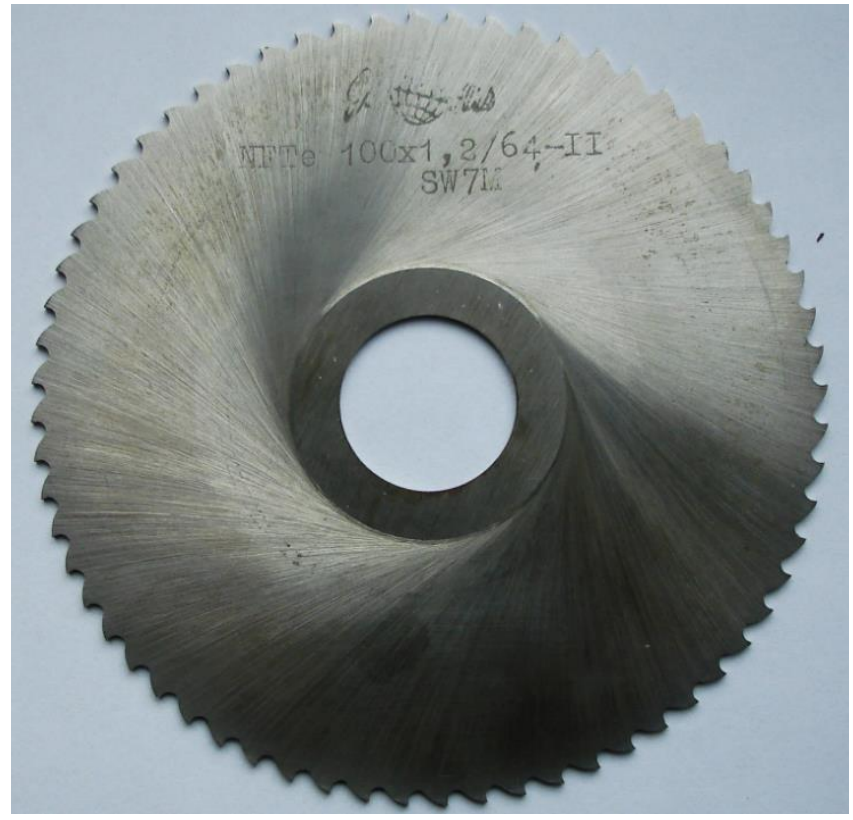

Fig. 6. NFTe 100x1.2/64-II circular slitting saw
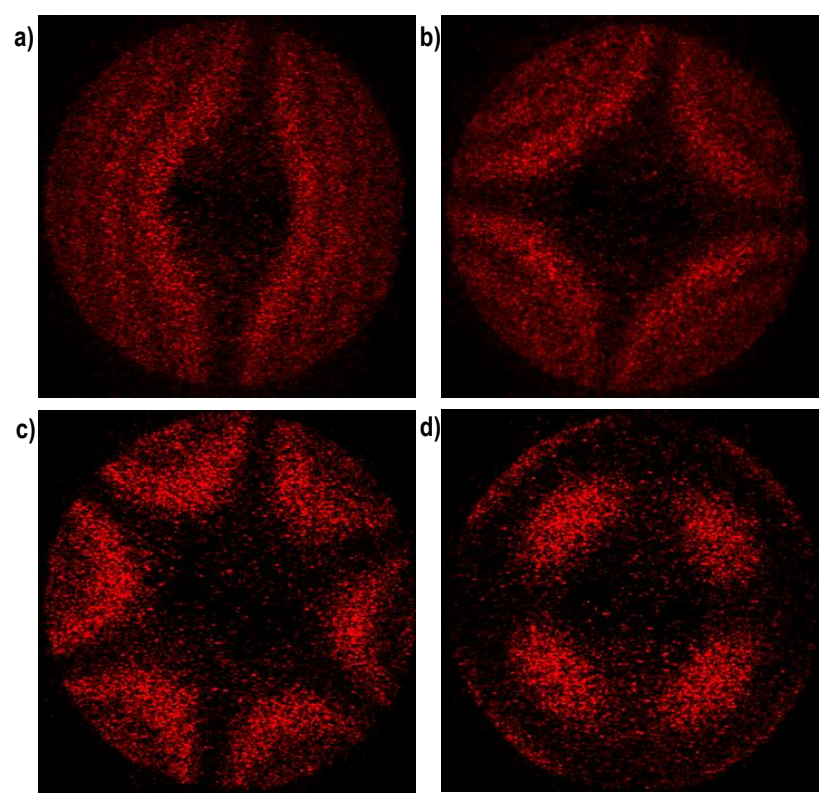

Fig. 7. Mode shapes of resonant vibrations of a circular slitting saw at different frequencies: a) $f=607 \mathrm{~Hz}$, b) $f=840 \mathrm{~Hz}$, c) $f=1495 \mathrm{~Hz}$, d) $f=5190 \mathrm{~Hz}$

Resonant frequencies $f_{i j}$ of the vibrating aluminum (AW $1050 \mathrm{~A}$ alloy) square plate were calculated using Eq. (13) for the values of plate material parameters: $E=70 \mathrm{GPa}, \rho=2700 \mathrm{~kg} / \mathrm{m}^{3}$, $v=0.3, a=0.09 \mathrm{~m}, h=0.002 \mathrm{~m}$. The values of square of dimensionless frequency parameter $\lambda_{i j}{ }^{2}$ for particular vibrating modes (Blevins, 2013) are shown in Tab. 1 and the results of calculations are compared with the experimental data. The values of relative error $\Delta \mathrm{f}_{\mathrm{ij}}$ are in a range of a few percent, therefore calculated and measured basic resonant frequencies are in good agreement.

Resonant frequencies $f_{i j}$ of the vibrating circular slitting saw were calculated using Eq. (13) for the values of parameters (SW7M - HS6-5-2 high-speed tool steel): $E=210 \mathrm{GPa}, \rho=7800$ $\mathrm{kg} / \mathrm{m}^{3}, v=0.3, \mathrm{a}=0.010 \mathrm{~m}, a / b=0.3$ (a and $\mathrm{b}$ are outer and inner diameters of the tool, respectively), $h=0.0012 \mathrm{~m}$. The values of 
square of dimensionless frequency parameter $\lambda_{i j}{ }^{2}$ for particular vibrating modes (Blevins, 2013) are shown in Tab. 2 and the results of calculations are compared with the experimental data.

Tab. 1. Analytically and experimentally determined resonant frequencies of a square Al plate $(x, y$ - vertical and horizontal direction, respectively)

\begin{tabular}{|c|c|c|c|c|}
\hline $\begin{array}{c}\lambda_{i j}{ }^{2} \\
\text { for a } \\
\text { vibratio- } \\
\text { nal mode }\end{array}$ & $\begin{array}{c}\text { Number } \\
(i, j) \\
\text { of half- } \\
\text { waves in } \\
x \text { and } y \\
\text { direction }\end{array}$ & $\begin{array}{c}\text { Resonant } \\
\text { frequency } \\
f_{i j \_a} \\
\text { (analytical) } \\
\text { [Hz] }\end{array}$ & $\begin{array}{c}\text { Resonant } \\
\text { frequency } \\
f_{i j \_} \\
\text {(experimental) } \\
\text { [Hz] }\end{array}$ & $\begin{array}{l}\text { Rela- } \\
\text { tive } \\
\text { error } \\
\Delta f_{i j} \\
{[\%]}\end{array}$ \\
\hline$\sum_{27.33}$ & $\begin{array}{l}i=1 \\
j=3\end{array}$ & 523 & 532 & 1.7 \\
\hline 21.43 & $\begin{array}{l}i=2 \\
j=1\end{array}$ & 410 & 390 & 5.1 \\
\hline
\end{tabular}

Tab. 2. Analytically and experimentally determined resonant frequencies of a circular saw

\begin{tabular}{|c|c|c|c|c|}
\hline $\begin{array}{c}\lambda_{i j^{2}} \\
\text { for a } \\
\text { vibratio- } \\
\text { nal mode }\end{array}$ & $\begin{array}{l}\text { Number } \\
(i, j) \\
\text { of nodal } \\
\text { diame- } \\
\text { ters and } \\
\text { nodal } \\
\text { circles }\end{array}$ & $\begin{array}{c}\text { Resonant } \\
\text { frequency } \\
f_{i j \_a} \\
\text { (analytical) } \\
{[\mathrm{Hz}]}\end{array}$ & $\begin{array}{c}\begin{array}{c}\text { Resonant } \\
\text { frequency } \\
f_{i j \_e}\end{array} \\
\text { (experimental) } \\
{[\mathrm{Hz}]}\end{array}$ & $\begin{array}{c}\text { Rela- } \\
\text { tive } \\
\text { error } \\
\Delta f_{i j} \\
\\
{[\%]}\end{array}$ \\
\hline & $\begin{array}{l}i=1 \\
j=0\end{array}$ & 745 & 607 & 22.7 \\
\hline & $\begin{array}{l}i=2 \\
j=0\end{array}$ & 936 & 840 & 11.4 \\
\hline & $\begin{array}{l}i=3 \\
j=0\end{array}$ & 1566 & 1495 & 4.7 \\
\hline
\end{tabular}

The values of relative error $\Delta f_{i j}$ are greater than in the case of the square plate experiment, particularly for lower resonant frequencies. For increasing values of a resonant frequency the experimental and theoretical results are in better agreement. In general, the analytical formula of Eq. (13) for resonant frequencies of a circular plate does not strictly apply to a cutting tool of a complex shape as a circular saw, due to differences in geometry between an analytical and the real object. The thickness of the saw used in the experiment was not uniform - it was decreasing radially from a value of $1.2 \mathrm{~mm}$ at the cutting edge to a value of $1.0 \mathrm{~mm}$ near a tool socket. A variable thickness is not included in analytical calculations. A more accurate computational method should be applied to precisely calculate the resonant frequency in this case. Resonance of the circular saw will occur when the frequency of excitation of the saw vibrations is equal to one of the experimentally determined resonant frequencies (Tab. 2). The frequency of excitation can be determined as the product of the rotational speed $n$ and the number $z$ of tool tips. Thus the values of rotational speed $n$ that should be avoided during machining using a circular saw with $z=64$ tips (Fig. 6) may be easily calculated for resonant frequencies shown in Tab. 2 $\left(n_{1}=9.5 \mathrm{~s}^{-1}, n_{2}=13.1 \mathrm{~s}^{-1}\right.$ and $n_{3}=23.4 \mathrm{~s}^{-1}$ for $f_{10} e=607 \mathrm{~Hz}$, $f_{20 \_}=840 \mathrm{~Hz}$ and $f_{30 \_}=1495 \mathrm{~Hz}$, respectively).

\section{SUMMARY}

Detailed knowledge on a range of undesirable frequencies resulting in high amplitude tool vibrations is an essential factor contributing to avoid the improper machining parameters. This issue is particularly important in a case of machining using tools susceptible to chatter diminishing the quality of a machined surface and generating excessive noise. Based on the research carried out, it appears that ESPI gives the opportunity to precisely determine the resonant frequencies of geometrically complex cutting tools. The experimental procedure allows for determining resonant frequencies with accuracy within a few $\mathrm{Hz}$. The important advantage of ESPI method is its simplicity which enables easy interpretation of the results. The conducted experiments showed the usability of this non-contact measurement method in a field of machining.

\section{REFERENCES}

1. Augulis L., Tamulevicius S., Augulis R., Bonneville J., Goudeau P., Templier C. (2004), Electronic speckle pattern interferometry for mechanical testing of thin film, Optics and Lasers in Engineering, 42, 1-8.

2. Beeck M.A., Hentschel W. (2000), Laser metrology - a diagnostic tool in automotive development processes, Optics and Lasers in Engineering, 34(2), 101-120.

3. Blevins R.D. (2016), Formulas for Dynamics, Acoustics and Vibration, John Wiley \& Sons, Ltd.

4. Chi-Hung H., Yu-Chih L., Chien-Ching M. (2004) Theoretical analysis and experimental measurement for resonant vibration of piezoceramic circular plates, IEEE Transactions On Ultrasonics, Ferroelectrics, And Frequency Control, 51(1), 12-24.

5. Foitzik A.H., Kaese W., Vogt T., Sommerer M., Arkhipov S. (2003), Static and Dynamic Characterization of MEMS via ESPI, International Journal Of Computational Engineering Science, 4(3), 467-470.

6. Fu G., Moosa A.G. (2002), An Optical Approach to Structural Displacement Measurement and Its Application, Journal of Engineering Mechanics, 128, 511-520.

7. Halama R., Hornacek L., Pecenka L., Krejsa M., Smach J. (2016), 3-D ESPI Measurements Applied to Selected Engineering Problems, Applied Mechanics and Materials, 827, 65-68.

8. Hild F., Roux S. (2006), Digital Image Correlation: from Displacement Measurement to Identification of Elastic Properties - a review, Strain, 42, 69-80.

9. Qin J., Gao Z., Wang X., Yang S. (2016), Three-Dimensional Continuous Displacement Measurement with Temporal Speckle Pattern Interferometry, Sensors, 16(12), 2020.

10. Richardson M.O.W., Zhang Z.Y., Wisheart M., Tyrer J.R. Petzing J. (1998), ESPI non-destructive testing of GRP composite materials containing impact damage, Composites Part A, 29A, 721-729.

11. Rothberg S.J., Bell J.R. (2004), On the application of laser vibrometry to translational and rotational vibration measurements on rotating shafts, Measurement, 35, 201-210. 
12. Tatar K., Gren P. (2008), Measurement of milling tool vibrations during cutting using laser vibrometry, International Journal of Machine Tools \& Manufacture, 48, 380-387.

13. Van der Auweraer H., Steinbichler H., Vanlanduit S., Haberstok C., Freymann R., Storer D., Linet V. (2002), Application of stroboscopic and pulsed-laser electronic speckle pattern interferometry (ESPI) to modal analysis problems, Measurements Science and Technology, 13, 451-463.

14. Yang L., Xie X., Zhu L., Wu S., Wang Y. (2014), Review of Electronic Speckle Pattern Interferometry (ESPI) for Three Dimensional Displacement Measurement, Chinese Journal Of Mechanical Engineering, 27(1), 1-13.

15. http://www.dantecdynamics.com, Dantec Dynamics, ESPI-Q100.

16. http://www.laserndt.com/technology/shearography.htm, Laser Technology Inc., Laser Shearography Technology.

17. http://resources.renishaw.com,

Brochure: XL-80 laser measurement system.

The work has been accomplished under the research project No. S/WM/2/2017 and S/WM/4/2017 financed by the Ministry of Science and Higher Education of Poland. 